\title{
Deep and surface hemodynamic signal from functional time resolved transcranial near infrared spectroscopy compared to skin flowmotion
}

\author{
Federico Aletti ${ }^{a, *, 1}$, Rebecca Re ${ }^{\mathrm{b}, 1}$, Vincenzo Pace ${ }^{\mathrm{a}, 1}$, Davide Contini ${ }^{\mathrm{b}}$, Erika Molteni ${ }^{\mathrm{a}}$, Sergio Cerutti ${ }^{\mathrm{a}}$, \\ Anna Maria Bianchi ${ }^{a}$, Alessandro Torricelli ${ }^{b}$, Lorenzo Spinelli ${ }^{c}$, Rinaldo Cubeddu ${ }^{\mathrm{b}}$, Giuseppe Baselli ${ }^{\mathrm{a}}$ \\ a Dipartimento di Bioingegneria IIT Unit, Politecnico di Milano, Milan, Italy \\ ${ }^{\mathrm{b}}$ Dipartimento di Fisica, Politecnico di Milano, Milan, Italy \\ ${ }^{\mathrm{c}}$ Istituto di Fotonica e Nanotecnologie, Consiglio Nazionale delle Ricerche, Milan, Italy
}

\section{A R T I C L E I N F O}

Keywords:

Cardiovascular regulation

Vasomotion

Valsalva maneuver

Time-resolved functional near infrared spectroscopy

Laser Doppler flowmetry

Prefrontal cortex hemodynamic activation

\begin{abstract}
A B S T R A C T
The potential disturbance in the prefrontal cortex hemodynamic signal measured by functional near infrared spectroscopy (NIRS), due to forehead skin flowmotion, detected by laser Doppler flowmetry, was investigated by a standard protocol of hemodynamic challenge by Valsalva maneuver, aimed at assessing and disentangling local regulatory responses in skin vasomotion and in cerebral perfusion in presence of a strong systemic drive, and to quantify the common information in the two signals. The deep cortical NIRS signal did not appear to be affected by surface vasomotor activity, and autoregulation dynamics were dominant with respect to autonomic control of circulation.
\end{abstract}

(c) 2011 Elsevier Ltd. All rights reserved.

\section{Introduction}

Functional Near Infrared Spectroscopy (fNIRS) is an emerging tool for non-invasive monitoring of changes of oxy- and deoxyhemoglobin $(\mathrm{Hb})$ concentrations in human tissues. Neurological applications of fNIRS have been widely exploited in the last decade [1-3]. The NIRS measurement is based on the diffusion of photons belonging to the near infrared region of the light spectrum through the skull and the different absorption spectra of oxy- and deoxy-Hb. The processing of the detected signals enables to extract the functional hemodynamic response, analog to the one measured in functional magnetic resonance imaging (fMRI). An open issue in the assessment of the relationship between the fNIRS signal and the functional response of the prefrontal cortex is represented by the need for an accurate and reliable methodology to disentangle global hemodynamic changes and cerebrovascular autoregulation from functional changes, being the latter related to cortical activations in response to proper stimuli or tasks and the former to cardiovascular (CV) autonomic and local regulation. Models based on time-resolved measures have been recently proposed to separate the confounding effects conveyed by skin flow from cortical activity, which can be traced back to early and late photons detected by fNIRS measurements, respectively [4,5,32].

\footnotetext{
*Corresponding author. Tel.: +390223993381; fax: + 390223993360 .

E-mail address: federico.aletti@polimi.it (F. Aletti).

1 These authors equally contributed to this paper.
}

However, a few assumptions underlying these models need more evidence in their support, as their validation has been mainly carried out by comparing the information extracted from fNIRS and fMRI measurements. An alternative option may entail the assessment of the optical response of different layers of the head: to fulfill this purpose, prefrontal cortex hemodynamics can be monitored by fNIRS, while flowmotion of the superficial layers of the forehead and temples skin can be measured by laser Doppler flowmetry (LDF) [6].

LDF provides continuous, non-invasive monitoring of microcirculatory blood flow dynamics, or vasomotion, or flowmotion, in the skin [7,8], investigating volumes of approximately $1 \mathrm{~mm}^{3}$, while penetrating to a depth of 1-2 $\mathrm{mm}$.

Stefanovska and colleagues [9] extracted and discussed the information on local haemodynamics conveyed by the LDF signal, pointing out the relationship with the classical CV spectral bands. Several studies [10-18] thoroughly investigated the information related to local hemodynamics and vasomotor regulation in conditions of severe stress of the whole CV system, characterized by the influence of strong systemic stimuli, which drive the main rhythms of hemodynamic variability through the autonomic nervous system (ANS).

Stauss et al. [19] specifically focused on the identification of sympathetic activity in the low frequency ( $L F \sim 0.1 \mathrm{~Hz}$ ) band of the power spectrum of LDF signals and interpreted their results in comparison with the classical power spectral analysis of systemic signals (ECG, respiration and arterial blood pressure), thus showing how skin vasomotion can be sympathetically induced and respond at a frequency around $0.1 \mathrm{~Hz}$ in humans. Such findings 
enable to discuss the frequency content of the LDF signal with respect to the $\mathrm{CV}$ variability spectrum; this may be preliminary to a deeper investigation of the relationship between local and systemic variabilities. In this regard, we recently proposed to tackle this problem by means of simulation studies [20].

On the basis of the aforementioned literature, the use of LDF to disentangle the contribution of peripheral autoregulation to hemodynamic variability from autonomic driven variability appears legitimate, therefore suggesting that LDF may help detecting disturbances carried by surface flowmotion to the functional component of fNIRS measurements.

To our knowledge, the simultaneous use of fNIRS and LDF devices was previously proposed in an invasive study on piglets [21]. Klaessens and coworkers chronically placed LDF probes on the dura to verify that the content of information in the fNIRS signal, is not disturbed by the noise due to local autoregulation, which contributes to the vasomotion signal detected by the LDF.

In this paper, the contemporaneous and non-invasive recording of LDF and fNIRS signals are proposed with the following goals: (1) to shed light on the physiological basis of the cross-talk phenomena between different $\mathrm{CV}$ control mechanisms active under stimulation of the ANS, of brain autoregulation, and of hemodynamic responses; (2) to estimate the confounding effects in the fNIRS time resolved signal due to superficial hemodynamics, which may improve the accuracy of fNIRS in describing cortical hemodynamic activation.

\section{Materials and methods}

Two experimental protocols were designed for the purpose of this paper. First, a preliminary protocol was necessary to determine if either device could be a source of noise and disturbance because of possible cross-talk phenomena between the two instruments. This testing phase of the experimental setup allowed to determine the optimal distance between the two LDF probes and the fNIRS optode. Then, an experimental protocol of autonomic stimulation for the disentanglement of hemodynamic effects due to cortex functional responses and surface skin flowmotion was completed.

Experimental recordings included a time resolved, frontopolar (Fp2), fNIRS, with an inter-fiber distance of $2 \mathrm{~cm}$ and two laser Doppler flowmetry (LDF) measurements. For further detail about the fNIRS instruments employed in this work see Re et al. [22].

\subsection{Phantom trials}

Phantom trials aimed at minimizing the optical interference between the two sources of signal, notably the disturbance produced by the LDF emitter on the fNIRS detectors. These trials allowed to determine that the optimal experimental set-up was achieved with the fNIRS optode placed in frontopolar (Fp2) position, with the two LDF probes placed on the left and right temples at a distance longer than $6 \mathrm{~cm}$ from the fNIRS optode, which was also equipped with two filters around the two wavelengths used in the fNIRS instrumentation ( 690 and $829 \mathrm{~nm}$ ) in order to filter out the LDF light; nonetheless, uncorrupted measures required the $6 \mathrm{~cm}$ minimum distance.

\subsection{Preliminary experiment}

Eight healthy volunteers (HV, age $25 \pm 2$ ), sitting comfortably in a quiet, conditioned (temperature $23 \pm 2{ }^{\circ} \mathrm{C}$ ), dimly lit room were required to perform a Valsalva Manoeuvre (VM) for a duration of $15 \mathrm{~s}$. All subjects, who voluntarily enrolled in the study after signing the informed consent authorization, were nonsmokers and had no history of cardiovascular or respiratory disorder. Alcohol, caffeine and any drugs were not taken for a half day before the experiments. The VM was preceded by a 10 min long pre-VM epoch of baseline recordings in conditions of spontaneous hemodynamic variability, and by a $10 \mathrm{~min}$ long recovery period after Valsalva, to assess the spontaneous vasomotor variability and recovery from the strong stimulation provided by VM, respectively.

The Valsalva maneuver is a widely used procedure for assessing the integrity of the ANS, in which a subject tries to exhale forcibly with a closed glottis so that no air exits through the mouth or nose as, for example, in strenuous coughing, straining during a bowel movement, or lifting a heavy weight. Specific changes occur in blood pressure and the rate and volume of blood returning to the heart, as the maneuver impedes venous return. Therefore, its main consequences on autonomic regulation consist in variations of perfusion and intrathoracic pressure, and in an increase of heart rate. The raise of intrathoracic pressure that occurs during the Valsalva maneuver elicits rapid changes in preload and afterload [23]. Heart rate and total peripheral resistance, which are increased during the execution of the maneuver, maintain their higher values in the immediate aftermath of it.

Since local haemodynamics are characterized by oscillations in the Very Low Frequency (VLF, $f<0.04 \mathrm{~Hz}$ ) band of the CV spectrum [9-18], experimental measurements with LDF have to be performed over sufficiently long intervals. A laser Doppler flowmeter ( $780 \mathrm{~nm} 1 \mathrm{~mW}$ ) (Perimed AB, Sweden), connected to a laptop equipped with a data acquisition system (Perimed Systems, Inc., Sweden) was used to record the temple skin flowmotion measured in relative units (Arbitrary Perfusion Units). Two coaxial probes (Perimed AB, Sweden) were placed on the right and left temples with double-sided tape. Prior to the beginning of the protocol, the subjects adapted to the environment where the experiment was carried out, sitting on an armchair for at least 10 min after placing the LDF and fNIRS probes on their head. Baseline recordings of spontaneous vasomotion, in conditions of spontaneous hemodynamic variability, were acquired for $10 \mathrm{~min}$ at rest. At the end of this baseline period all subjects were asked to execute a forced expiratory maneuver (VM) for $15 \mathrm{~s}$. Then recovery baseline recordings followed for $10 \mathrm{~min}$.

The rationale of the preliminary experiment was to validate that two LDF measurements, apart from the site where the fNIRS probe is placed, are sufficiently correlated to be assumed as acceptable surrogates of a LDF measurement in the same location of the fNIRS measure, which was not possible due to optic crosstalk between the two devices, and to the disturbance on fNIRS measurements by LDF. The homogeneity of LDF measurements in different sites of human face was to be tested because human face is an heterogeneous microvascular region: angiographic characteristics of deep horizontal sub-dermal plexus, endothelial and vascular smooth muscle cell heterogeneity, and plasticity of the microvasculature, autonomic asymmetry and facial neuropsychological asymmetry are possible causes of microvascular asymmetry [24].

Thus, the preliminary experiment was necessary to assess, by means of correlation analysis, if perfusion dynamics acquired on the left and right temples may be averaged in order to reduce the amount of data during simultaneous acquisition with fNIRS signals in the main experiment. The good correlation obtained in the preliminary experiment subsequently enabled to average the two laser Doppler signals in the main experiment in order to reduce redundant data (simultaneous acquisition of fNIRS signals)

The coherence between the two distant LDF signals was computed from spontaneous variability recordings, during VM and during recovery from it. This was considered a pre-requisite for the two LDF signals to provide with a sufficiently reliable measure of skin flow to the forehead and temples, representative 
of the surface hemodynamic signal in correspondence of the prefrontal cortex whose perfusion can be detected by a fNIRS device.

\subsection{Autonomic stimulation experiment}

The fNIRS optode was placed on the forehead, slightly to the right of the frontal suture, in Fp2 position, while the LDF probes were placed on the left and right temples at a distance longer than $6 \mathrm{~cm}$ from the fNIRS optode for the reasons illustrated in the Section 2.1.

The experimental set-up and the protocol were the same of the preliminary protocol (10 min of spontaneous variability recordings, $15 \mathrm{~s}$ of forced expiratory Valsalva maneuver, $10 \mathrm{~min}$ of recovery to baseline).

Thirteen healthy volunteers (age $28 \pm 2$ ) were enrolled and gave their informed consent to participate in the study.

\subsection{Signal processing and analysis for the main experiment}

Artifact free, approximately 5 min long stationary segments were selected in pre-VM and post-VM epochs of the acquired data for both LDF and fNIRS signals.

(i) fNIRS pre-processing: fNIRS signals were recorded at $1 \mathrm{~Hz}$ to improve the signal to noise ratio. Analyzing the first part (early gate) of the time resolved photon time of flight distribution, we consider photons that travel few hundreds of picoseconds into the tissue and, thus, have a low probability to reach the brain cortex, while analyzing the tail of the distribution (late gate) we consider photons with a higher probability to go deep into the tissue and bring information about brain activity. Thus, exploiting the depth information, naturally encoded into photons arrival time, it is possible to discriminate between extra- and intra-cerebral hemodynamic signals [32].

The same pre-processing procedures were applied to both extra- and intracerebral (early and late gate, respectively) signals, either referred to oxy- and deoxy-hemoglobins. In the following, the signals will be referred to as oxyHb extra, deoxyHb extra, oxyHb intra, and deoxyHb intra.

(ii) LDF pre-processing: LDF signals were acquired at $32 \mathrm{~Hz}$, then time series were extracted and downsampled to $4 \mathrm{~Hz}$, by means of an antialising low-pass filter, in order to limit the bandwidth to the regulatory mechanisms of interest, from very slow local vasomotor phenomena related to vascular autoregulation up to faster respiratory and heartbeat frequencies. In addition, time series derived from the right and left LDF raw measurements were averaged.

LDF signal was first filtered by a moving average to remove any residual low frequency trends, which might introduce spurious peaks in the power spectrum.

(iii) Time domain analysis: Time domain analysis entailed the computation of cross-correlation functions between the averaged LDF signal and oxyHb extra, deoxyHb extra, oxyHb intra, and deoxyHB intra in three different epochs: preValsalva (1200 samples), during Valsalva (50 samples) and post-Valsalva (1200 samples).

(iv) Frequency domain analysis: Power spectra of time series before and after VM were estimated via autoregressive (AR) parametric model estimation, and also computed by nonparametric approaches (Welch's modified periodogram).

While AR spectral estimation provided with good results with LDF time series, severe limitations were found with fNIRS, due to high levels of baseline drifts. For this reason the analysis was repeated by means of Welch's periodogram and results presented in the following are referred to this spectral computation technique.

Original signals were split into shorter segments, partially overlapping (overlap of 50\%). A data window (Hamming window of 80 samples, $f_{s}=1 \mathrm{~Hz}$ ) was applied to each stationary segment (pre-VM, post-VM) and a modified periodogram was computed. The use of the Hamming window was aimed at improving the spectral resolution by reducing the effect of lateral lobes. Finally power spectral density (PSD) estimates of all segments were averaged.

Power in the VLF band (frequencies $(f)<0.04 \mathrm{~Hz}$ ), in the autonomic bands (LF, $0.04 \mathrm{~Hz}<f<0.15 \mathrm{~Hz}$; high frequency, $\mathrm{HF}$, $0.15<f<0.4 \mathrm{~Hz}$ ), and in the band where the dominating phenomenon is the pulse $(0.8<f<2 \mathrm{~Hz}$ was computed [25].

\subsection{Statistical analysis}

All statistics were performed using $R$ statistical software and Matlab Statistical Toolbox.

For the time interval preceding VM, the statistical analysis was carried out by applying parametric $t$-paired test to correlation data of the paired LDF channels; a Wilcoxon rank sum nonparametric test was used to assess significant differences between LDF and fNIRS data.

Differences between two groups (e.g. pre-VM vs. VM; post-VM vs. VM and pre-VM vs. post-VM) were analyzed using paired 2-tailed Student's $t$ test if Kolmogorov-Smirnov null hypothesis of normal distribution could not be rejected. The level of significance was set to $p=0.05$. Normalized correlation coefficients between LDF and fNIRS during VM and power contents in the VLF and "autonomic" band were instead compared using Wilcoxon sum rank nonparametric test in order to get more robust statistics being the population not normally distributed (KolmogorovSmirnov, $p<0.05$ ).

Regression analysis was applied to quantify the correlation between the spectral power of LDF and fNIRS time series in the VLF band and in the autonomic band (LF+HF). Also, crosscorrelation analysis was carried out to assess the correlation between VLF, LF, and HF normalized powers in the spectra of the left and right temples laser Doppler flowmotion signals.

Boxplots were derived to compare medians and averages of different populations through paired Student $t$-test.

Wilcoxon sum-rank nonparametric test was applied to the second phase-protocol data.

\section{Results}

\subsection{Preliminary experiment}

Correlation analysis between LDF channels (left temple, right temple) before VM provided with the correlation coefficient range of microcirculation perfusion at rest $(0.65 \pm 0.85)$. During recovery following $\mathrm{VM}$, the coefficient decreased to $(0.45 \pm 0.85)$ (Fig. 1). Pre-VM $r$-coefficient values were higher than post-VM in 7 subjects out of $8(p<0.01)$ (Table 1$)$. During VM, higher values of correlation between the two LDF channels were found $(0.96 \pm 0.02, p<0.001)$.

\subsection{Main experiment}

All 13 subjects enrolled in the main experiment (simultaneous recording of LDF signals on the left and right temples, fNIRS recording on the forehead, in Fp2 position) displayed similar responses to $\mathrm{VM}$, with particularly distinguishable and repeatable 
patterns in the averaged LDF signal and in the surface oxy-Hb dynamics (respectively, red and black tracings in Fig. 2).

Both transients showed an undershoot during the deep inspiration preparing the Valsalva onset and a marked overshoot during the forced expiration. After VM ending (15 s after the onset), surface oxy-Hb concentration fell more rapidly ( $\sim 13 \mathrm{~s}$ ) than the LDF signal $(\sim 18 \mathrm{~s})$.

Intra cerebral fNIRS signals, calculated from the late photons measurements, did not show the fast rising spike, which appears at the onset of VM in LDF and superficial oxy-Hb during the forced expiration (Fig. 3).

High correlation $(r>0.9)$ was found during VM between LDF and all fNIRS signals; however, after scaling it (Fig. 4) by fNIRS signal rms (i.e., fNIRS rms correlated to LDF), significantly higher

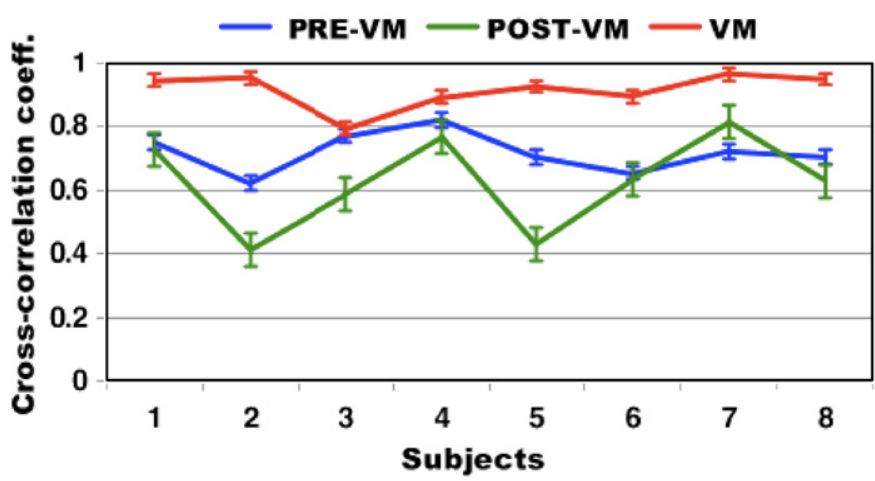

Fig. 1. Cross-correlation coefficients between left and right-temple skin flow signals in pre-VM, during Valsava, and post-VM periods in the 8 subjects participating in the preliminary protocol. (Error bars indicate standard error).

Table 1

Coefficients of correlation between LDF signals recorded from left and right temples. During VM correlation is significant in 7 out of 8 subjects $(p<0.01)$.

\begin{tabular}{llll}
\hline Subject \# & Pre-VM $(p$-value $)$ & VM $(p$-value $)$ & Post-VM $(p$-value $)$ \\
\hline 1 & $0.75 p<0.01$ & $0.94 p<0.001$ & $0.73 p<0.01$ \\
2 & $0.62 p=0.08^{\mathrm{a}}$ & $0.95 p<0.001$ & $0.41 p=0.12^{\mathrm{a}}$ \\
3 & $0.77 p<0.01$ & $0.79 p=0.07^{\mathrm{a}}$ & $0.59 p=0.09^{\mathrm{a}}$ \\
4 & $0.82 p<0.01$ & $0.89 p<0.01$ & $0.77 p<0.05$ \\
5 & $0.70 p<0.01$ & $0.92 p<0.001$ & $0.43 p=0.07^{\mathrm{a}}$ \\
6 & $0.65 p=0.05$ & $0.89 p<0.01$ & $0.63 p<0.05$ \\
7 & $0.72 p<0.01$ & $0.96 p<0.001$ & $0.81 p<0.01$ \\
8 & $0.70 p<0.01$ & $0.95 p<0.001$ & $0.63 p=0.05$ \\
\hline
\end{tabular}

${ }^{a}$ Not significant correlations.

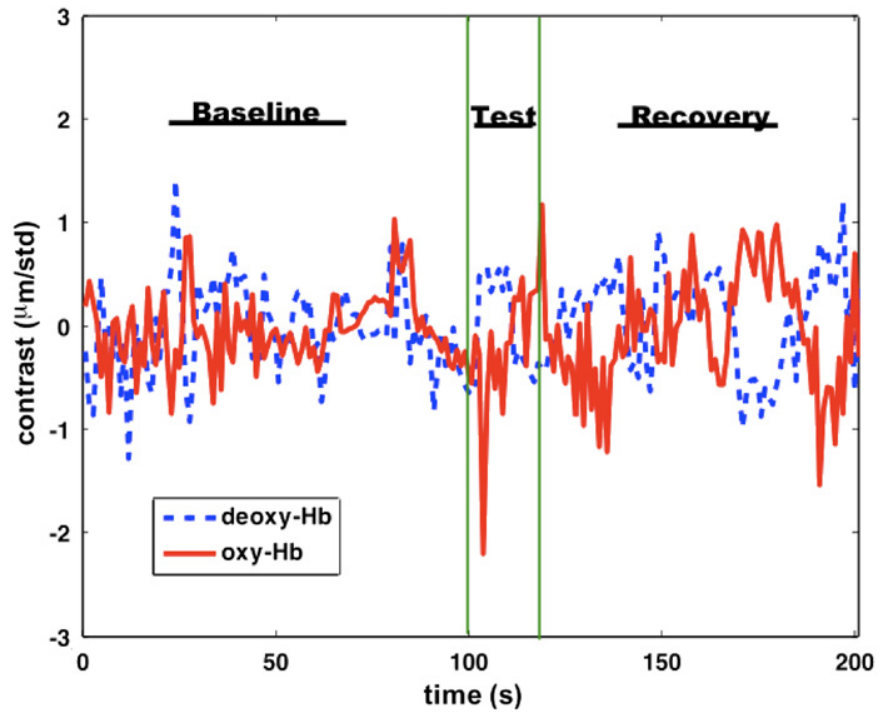

Fig. 3. Grand average of fNIRS cortical oxy-Hb (red) and deoxy-Hb (blue) measurements, referred to late photons measures of intracerebral haemodynamics. (For interpretation of the references to color in this figure legend, the reader is referred to the web version of this article.)

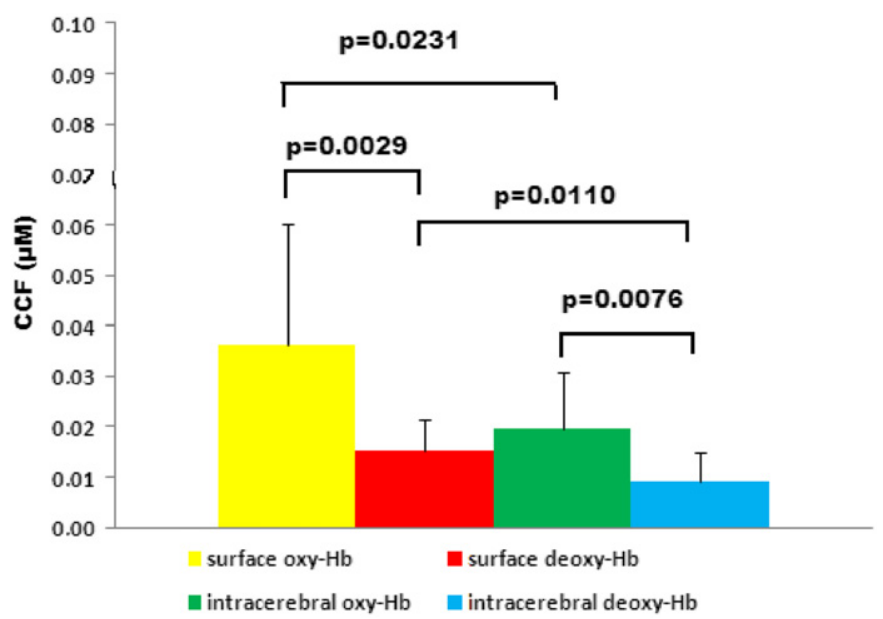

Fig. 4. Cross-correlation coefficients between average LDF and fNIRS recordings, rescaled by rms of surface and cortex oxy-Hb and deoxy-Hb. Data are mean \pm SD. Correlation between LDF and surface oxy-Hb value is significantly higher than between LDF and deoxy-Hb value $(p=0.0029)$; correlation between LDF and extracerebral oxy-Hb values are significantly higher than intracerebral oxy-Hb values $(p=0.023)$.

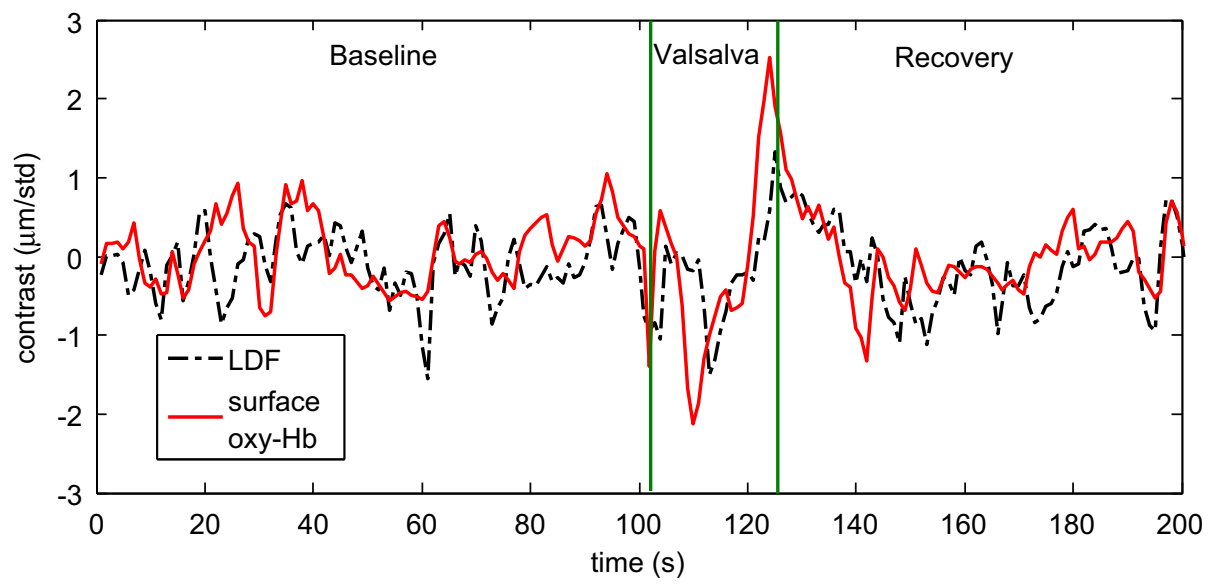

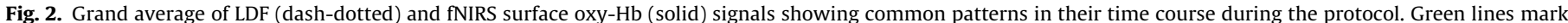
VM onset and offset. (For interpretation of the references to color in this figure legend, the reader is referred to the web version of this article.) 
values $(0.04 \pm 0.010, p=0.02$ surface oxy-Hb; $0.01 \pm 0.006, p=0.01$ surface deoxy-Hb) were obtained for surface signals compared to cortical ones $(0.02 \pm 0.008$ cortex oxy-Hb; $0.009 \pm 0.0015$ cortex deoxy-Hb). Similarly, higher $r$-coefficients were found for oxy-Hb compared to deoxy-Hb. For details on correlation coefficients, see Table 2.

During pre and post-VM, the spectral analysis of LDF and fNIRS time-series revealed the presence of a large VLF component and significant activity in the LF (vasomotor) and HF (respiratory) band (Fig. 5). Due to the relatively low spontaneous variability of the fNIRS signal, and to possible entrainment phenomena occurring when respiratory frequencies of subjects slowed and approached the $0.15 \mathrm{~Hz}$ (which may have occurred because of the length of the protocol, and other experimental conditions, such as dim light in the room, comfortable position, etc.) limit separating LF and HF bands, the LF and HF bands were lumped together and taken into consideration as one band, representative of autonomic control of hemodynamics. This was also justified by the fact that either in pre-VM and post-VM epochs, both LDF and fNIRS displayed VLF and LF+HF activity, but low correlations $(0.1<r<0.2)$ were found in both bands between fNIRS and LDF signals.

Power coherence calculation confirmed VLF band-related power to be the main common power component of surface skin flowmotion signal detected by LDF and extracerebral oxy-HB obtained through fNIRS, both before and after VM (Fig. 6).

The power in the VLF and LF + HF bands did not vary significantly following the Valsalva maneuver in either LDF or extracerebral oxy-Hb signals (Figs. 7 and 8). The preponderant power content in the VLF band was consistent with the typically

Table 2

Correlation values between averaged LDF signal and surface oxy (deoxy)-hemoglobin and cortical oxy (deoxy)-hemoglobin.

\begin{tabular}{|c|c|c|c|c|}
\hline Subject \# & LDF_deoxyHb extra & LDF_oxyHb extra & LDF_-deoxyHb intra & LDF_oxyHb intra \\
\hline 1 & $0.02(p=0.021)$ & $0.07(p<0.001)$ & $0.01(p=0.038)$ & $0.04(p<0.001)$ \\
\hline 2 & $0.005(p=0.041)$ & $0.03(p=0.023)$ & $0.008(p=0.04)$ & $0.01(p=0.025)$ \\
\hline 3 & $0.01(p=0.030)$ & $0.05(p<0.001)$ & $0.007(p=0.05)$ & $0.03(p=0.007)$ \\
\hline 4 & $0.0015(p=0.065)^{\mathrm{a}}$ & $0.02(p=0.06)$ & $0.0086(p=0.819)^{\mathrm{a}}$ & $0.02(p=0.884)^{\mathrm{a}}$ \\
\hline 5 & $0.0017(p<0.01)$ & $0.09(p<0.001)$ & $0.0065(p=0.124)^{\mathrm{a}}$ & $0.015(p=0.006)$ \\
\hline 6 & $0.008(p=0.366)^{\mathrm{a}}$ & $0.02(p=0.008)$ & $0.009(p=0.05)$ & $0.005(p=0.78)^{a}$ \\
\hline 7 & $0.025(p=0.01)$ & $0.01(p=0.714)^{\mathrm{a}}$ & $0.003(p=0.8)^{\mathrm{a}}$ & $0.01(p=0.960)^{\mathrm{a}}$ \\
\hline 8 & $0.021(p=0.02)$ & $0.04(p=0.005)$ & $0.025(p=0.65)^{\mathrm{a}}$ & $0.013(p=0.025)$ \\
\hline 9 & $0.017(p=0.018)$ & $0.03(p=0.398)^{\mathrm{a}}$ & $0.009(p=0.034)$ & $0.02(p=0.212)^{\mathrm{a}}$ \\
\hline 10 & $0.019(p=0.04)$ & $0.02(p<0.001)$ & $0.0075(p=0.48)$ & $0.03(p=0.017)$ \\
\hline 11 & $0.013(p=0.23)^{\mathrm{a}}$ & $0.016(p=0.832)^{\mathrm{a}}$ & $0.004(p=0.9)^{\mathrm{a}}$ & $0.036(p<0.001)$ \\
\hline 12 & $0.022(p=0.156)^{\mathrm{a}}$ & $0.04(p=0.006)$ & $0.0023(p=0.96)^{\mathrm{a}}$ & $0.019(p=0.142)^{\mathbf{a}}$ \\
\hline 13 & $0.009(p=0.121)^{\mathrm{a}}$ & $0.037(p=0.03)$ & $0.016(p=0.04)$ & $0.005(p=0.556)^{a}$ \\
\hline
\end{tabular}

a $p$-Values $>0.05$ (not significant values of correlation). In 10 out of 13 subjects, correlation coefficients between laser Doppler and extracerebral oxy-Hb are significant $(p$-value $<0.05)$

a

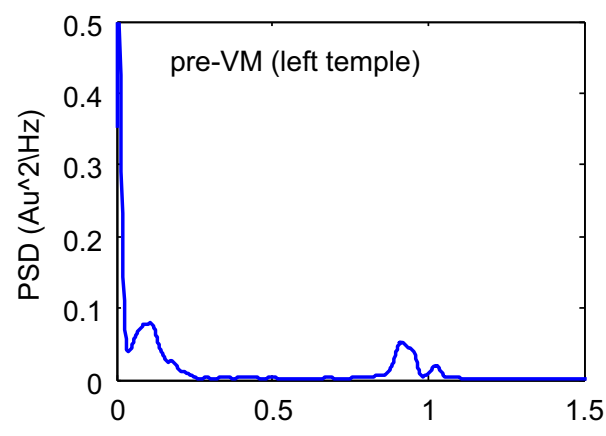

C

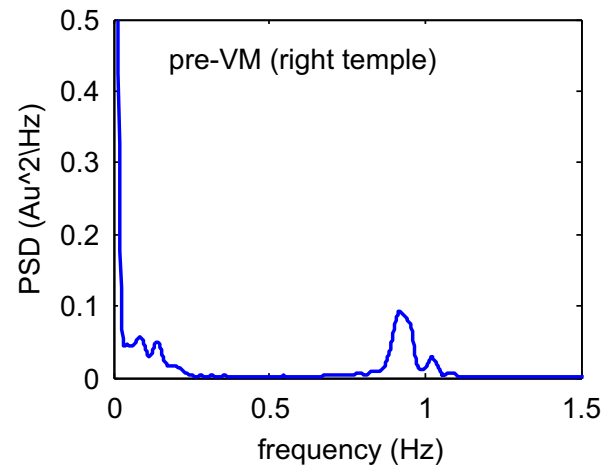

b

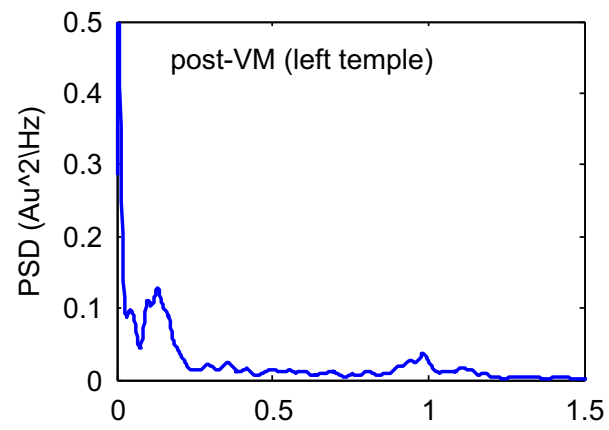

d

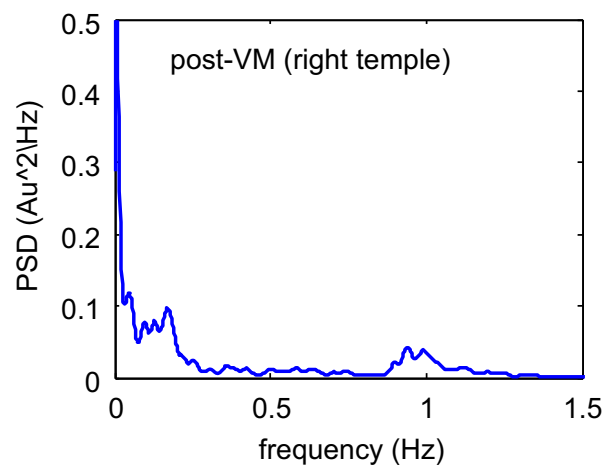

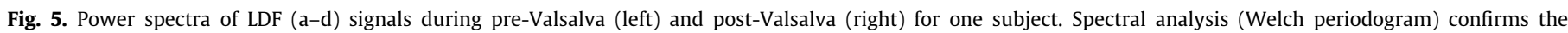
overlapping of LF and HF bands. 


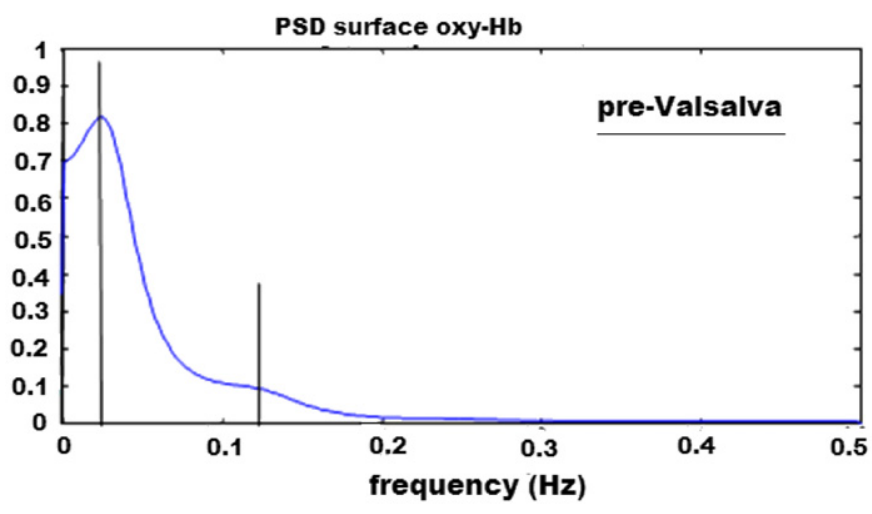

Fig. 6. Power spectrum of oxy-Hb extracerebral NIRS signal during pre-Valsalva for one subject. Spectral analysis shows that the power component in HF band is not significant in fNIRS signals.

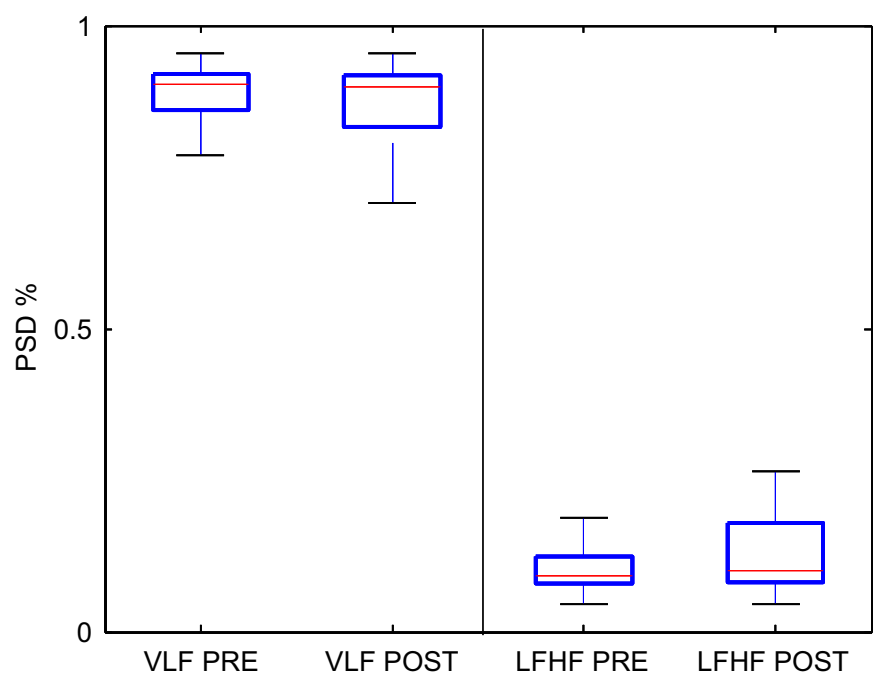

Fig. 7. Box-plot of total VLF (left) and LF + HF (right) power of LDF before and after VM.

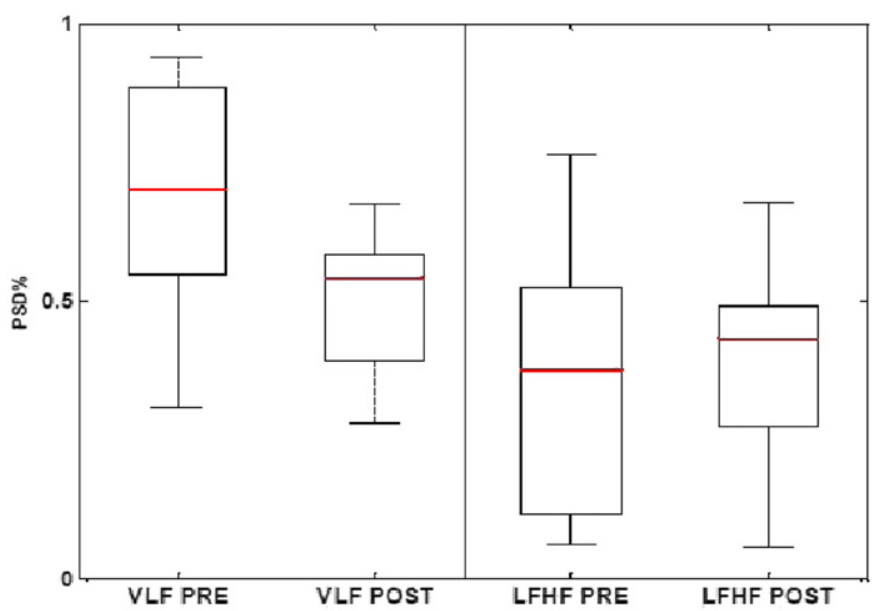

Fig. 8. Box-plot of total VLF (left) and $L F+H F$ (right) power of superficial oxy-Hb before and after VM.

slow rhythms of peripheral microvascular hemodynamics [9-18], which characterize the vasomotion phenomena detected by the LDF signal. Values of VLF and LF + HF power components assessed
Table 3

Values of VLF and LF+HF components assessed on LDF and fNIRS signals (mean \pm std).

\begin{tabular}{llllll}
\hline & \multicolumn{3}{l}{ Pre-VM } & & \multicolumn{2}{l}{ Post-VM } \\
\cline { 2 - 3 } \cline { 5 - 6 } & VLF & LF + HF & \multicolumn{2}{l}{ VLF } & \multicolumn{1}{l}{ LF + HF } \\
\hline LDF & $0.889 \pm 0.050$ & $0.101 \pm 0.036$ & & $0.868 \pm 0.075$ & $0.131 \pm 0.066$ \\
Deoxy-Hb extra & $0.711 \pm 0.160$ & $0.289 \pm 0.192$ & & $0.609 \pm 0.157$ & $0.391 \pm 0.136$ \\
Oxy-Hb extra & $0.648 \pm 0.155$ & $0.351 \pm 0.159$ & & $0.635 \pm 0.140$ & $0.365 \pm 0.142$ \\
Deoxy-Hb intra & $0.750 \pm 0.127$ & $0.249 \pm 0.118$ & & $0.739 \pm 0.101$ & $0.264 \pm 0.096$ \\
Deoxy-Hb intra & $0.770 \pm 0.120$ & $0.232 \pm 0.119$ & $0.756 \pm 0.117$ & $0.246 \pm 0.115$ \\
\hline
\end{tabular}

on LDF and fNIRS signals (mean \pm std) are reported in detail in Table 3.

\section{Discussion}

The phantom trials and the preliminary experiment enabled to analyze the cross-talk between the two instruments before the main experimental protocol. In particular, the phantom trials determined that the optimal set-up would entail placing the two LDF probes on the opposite sides of the forehead, with the fNIRS optode placed in correspondence to the medial portion of the frontal lobe, because of the different power emitted by the laser Doppler flowmeter and the near-infrared spectroscope.

Subsequently, the preliminary test on the information carried by the two LDF signals collected on the two temples showed that vasomotion related dynamics detected by the two probes were highly correlated and comparable. Given that it would be virtually impossible to thoroughly map the entire forehead by an empiric approach aimed at finding potential discrepancies in local vasomotor responses [24], these results basically revealed the affinity in the information conveyed by the two LDF recordings, thus suggesting that typical vasomotor and autoregulation dynamics in the two measurement sites could be considered homogeneous. This preliminary experiment also showed the effects of the strong hemodynamic perturbation induced by VM: (a) a sharp synchronization of central and peripheral oscillations during the forced expiration, indicated by the high value of correlation between the two LDF channels; and (b) a marked desynchronization following $\mathrm{VM}$, during recovery.

The main experiment investigated the potential correlation between fNIRS extracerebral signals and surface skin flowomotion detected by LDF.

The comparable patterns shown by both LDF and extracerebral fNIRS in the time domain appeared consistent with previous results reported by Tsuji et al. [26], and Brady et al. [27], who respectively found a peak of coherence between fNIRS signal and arterial blood pressure in neonates and between fNIRS and LDF in piglets, at frequencies lower than $0.04 \mathrm{~Hz}$.

Our results also showed consistency with Themelis et al. [28], who observed good correlation between the information captured by the LDF and the time continuous fNIRS signal during the evaluation of the scalp influence in the measurement of arterial oscillations originating in the brains of piglets. In addition, the normalized cross-correlation coefficients (Fig. 5) further emphasized the relationship between surface hemodynamics related to local flowmotion and the extracerebral component of the fNIRS data.

The reported findings imply that the information conveyed by LDF skin vasomotion measurements support the validation of the statistical hypothesis underlying the definition of the "extracerebral signal" detected by the time-resolved fNIRS instrument, and assumed to be representative of the extra cortical areas of the 
frontal skull. This evidence strengthens that the model-based separation of time-resolved NIRS early (superficial) and late (deep) photons is able to cancel, or at least attenuate, the surface confounding effects.

Although one of the limitations of the current protocol might be the ability of the LDF to investigate a volume of the forehead comparable, in depth, to the surface portion that is included in the extracerebral fNIRS signal, it was also clearly emphasized that the comparable patterns and the cross-correlation between the LDF grand average and the extracerebral fNIRS supported the hypothesis that early photons dynamics are strongly influenced by surface skin vasomotion. This may partly depend on hemodynamic changes unrelated to cortical variations in blood flow. The ensuing interpretation, thus, is that this dependency could be ascribed to systemic changes, which are particularly significant under the drive of strong hemodynamic provocations such as a Valsalva maneuver.

Obrig et al. [29] showed that systemic changes may appear in the extracerebral compartment and affect fNIRS measurements. On the other hand, Stefanovska et al. [9] showed that skin blood vasomotion is subject to systemic drives and stimuli. Our results were consistent with these previous reports, although local flow dynamics basically reflect the effect of vasomotion on peripheral resistance and flow in a much limited peripheral district of the arterial tree. Still, even if microcirculation is not innervated, the drive of the beating heart, of respiration, and of global control of circulation is transmitted to the peripheries of the arterial tree by the pulsating arterial wave, whose dynamic features are largely buffered by peripheral compliances.

\section{Summary}

This study demonstrated the feasibility of simultaneous fNIRS and LDF measurements and confirmed the effectiveness of time resolved fNIRS in separating deep from surface signals during provocative maneuvers like a Valsalva maneuver. Our experimental and analytical approach proposed a simple way to validate the interpretation of time-resolved fNIRS signal as representative of functional and hemodynamic cortical activation.

One of the issues which remains to be addressed is whether autonomic control [30] affects deep cortical hemodynamics more than simple systemic provocative tests, such as Valsalva. Further experiments, including other autonomic tests, might shed light on the difference in variability between surface and cortex fNIRS signals.

Considering the potential significance of fNIRS in portraying the functional activation of the prefrontal cortex, it might also be useful to further investigate the relationship between surface flowmotion and deep recordings in presence of simple functional tasks [31], such as finger tapping; in this case, the separation of the relevant confounding effects, which can be elicited by the likely side effects due to a contemporaneous autonomic activation whose role is still be to clarified, might prove instrumental in correctly characterizing the functional content of deep fNIRS as a reliable measurement of cortical activation.

\section{Conflict of interest statement}

None declared

\section{Acknowledgments}

This research has been funded by the European Community's Seventh Framework Program (FP7 2007-2013) under the grant agreement n. HEALTH-F5-2008-201076 nEUROpt. We would also like to thank Perimed Italia Srl, Cuggiono, Italy.

\section{References}

[1] A. Villringer, Functional neuroimaging. Optical approaches, 1-18, Adv. Exp Med Biol (1997).

[2] M. Calderon-Arnulph, A. Alaraj, K. Slavin, Near Infrared technology in neuroscience: past, present and future, Neurol. Res. (2009) 605-614.

[3] M. Wolf, M. Ferrari, V. Quaresima, Progress of near-infrared spectroscopy and topography for brain and muscle clinical application, J. Biomed. Opt. (2007) 12-14.

[4] A. Liebert, H. Wabnitz, J. Steinbrink, H. Orbig, M. Möller, R. Macdonald, A. Villringer, H. Rinneberg, Timeresolved multidistance near-infrared spectroscopy of the adult head: intracerebral and extracerebral absorption changes from moments of distribution of times of flight of photons, Appl. Opt. (2004) 3037-3047.

[5] D. Contini, A. Torricelli, A. Pifferi, L. Spinelli, F. Paglia, R. Cubeddu, A multichannel time-resolved system for functional near infrared spectroscopy, Opt Expr 14 (2006) 5418-5432.

[6] A.P. Shepherd, P.Å. Öberg, Laser Doppler flowmetry (1990).

[7] H. Peng, V. Matchkov, A. Ivarsen, C. Aalkjaer, H. Nilsson, Hypothesis for the initiation of vasomotion, Circ. Res. 88 (2001) 810-815.

[8] H. Nilsson, C. Aalkjaer, Vasomotion: mechanisms and physiological importance, Mol. Interv. 3 (2) (2003) 79-89.

[9] A. Stefanovska, M. Bracic, H.D. Kvernmo, Wavelet analysis of oscillations in the peripheral blood circulation measured by laser Doppler technique, IEEE Trans. Biomed. Med. Eng. (1999) 1230-1239.

[10] H.D. Kvernmo, A. Stefanovska, M. Bracic, K.A. Kirkeboen, K. Kvernebol, Spectral analysis of the Laser Doppler perfusion signal in human skin before and after exercice, Microvasc. Res. 56 (1998) 173-182.

[11] E.H. Serné, R.G. Ijzerman, R.O.B. Gans, R. Nijveldt, G. de Vries, R. Evertz, A.J.M. Donker, C.D.A. Stehouwer, Direct evidence for insulin-induced capillary recruitment in skin of healthy subjects during physiological hyperinsulinemia, Diabetes 51 (2002) 1515-1522.

[12] K. Urbancic-Rovan, A. Stefanosvka, A. Bernjak, K. Azman-Juvan, A. Kocijancic, Skin blood flow in the upper and lower extremities of diabetic patients with and without autonomic neuropathy, J. Vasc. Res. 41 (2004) 535-545.

[13] M. Rossi, R. Ricco, A. Carpi, Spectral analysis of skin Laser Doppler blood perfusion signal during cutaneous hyperemia in response to acetylcholine iontophoresis and ischemia in normal subjects, Clin. Hemorheol. Microcirc. 31 (2004) 303-310.

[14] M. Rossi, S. Maurizio, A. Carpi, Skin blood flowmotion response to insulin iontophoresis in normal subjects, Microvasc. Res. 70 (2005) 17-22.

[15] M. Rossi, S. Bertuglia, M. Varanini, A. Giusti, G. Santoro, A. Carpi, Generalised wavelet analysis of cutaneous flowmotion during post-occlusive reactive hyperemia in patients with peripheral arterial obstructive disease, Biomed. Pharmacother. 59 (2005) 233-239.

[16] M. Rossi, A. Carpi, C. Di Maria, F. Galetta, G. Santoro, Spectral analysis of Laser Doppler skin blood flow oscillations in human essential arterial hypertension, Microvasc. Res. 72 (2006) 34-41.

[17] P. Kvandal, S.A. Landsverk, A. Bernjak, A. Stefanovska, H.D. Kvernmo, K.A. Kirkeboen, Low-frequency oscillations of the laser Doppler perfusion signal in human skin, Microvasc. Res. 72 (3) (2006) 120-127.

[18] S.A. Landsverk, P. Kvandal, A. Bernjak, A. Stefanovska, K.A. Kirkeboen, The effects of general anesthesia on human skin microcirculation evaluated by wavelet transform, Anesth. Analg. 105 (4) (2007) 1012-1019.

[19] H.M. Stauss, E.A. Anderson, W.G. Haynes, K.C. Kregel, Frequency response characteristics of sympathetically mediated vasomotor waves in humans, Am. J. Physiol. 274 (1998) H1277-H1283.

[20] F. Aletti, E. Lanzarone, M.L. Costantino, G. Baselli, Simulation study of autoregulation responses of peripheral circulation to systemic pulsatility, Nonlinear Biomed. Phys. 24;3(1):7 (2009).

[21] J.H.G.M. Klaessens, R.G.M. Kolkman, J.C.W. Hopman, E. Hondebrink K.D. Liem, W. Steenbergen, F.F.M. de Mul, J.M. Thijssen, Monitoring cerebral perfusion using near-infrared spectroscopy and laser Doppler flowmetry, Physiol. Meas. 24 (4) (2003) 35-40.

[22] R. Re, D. Contini, M. Caffini, R. Cubeddu, L. Spinelli, A. Torricelli, A compact time-resolved system for near infrared spectroscopy based on wavelength space multiplexing, Rev. Sci. Instrum. 81 (2010) 113101.

[23] C.J. Porth, V.S. Bamrah, F.E. Tristani, J.J. Smith, The Valsalva maneuver: mechanisms and clinical implications, Heart Lung. 13 (5) (1984) 507-518.

[24] M. Benedicic, A. Bernjak, A. Stefanovska, R. Bosnjak, Continuous wavelet transform of laser-Doppler signals from facial microcirculation reveals vasomotion asymmetry, Microvasc. Res. 74 (1) (2007) 45-50.

[25] Eur. Heart J. 17 (3) (1996) 354-381 Mar.

[26] M. Tsuji, M.J.P. Saul, A. du Plessis, E. Eichenwald, J. Sobh, R. Crocker, J.J. Volpe, Cerebral intravascular oxygenation correlates with mean arterial pressure in critically ill premature infants, Pediatrics 106 (2000) 625-632.

[27] K.M. Brady, J.K. Lee, K.K. Kibler, P. Smielewski, M. Czosnyka, R.B. Easley, R.C. Koehler, D.C. Shaffner, Continuous time-domain analysis of cerebrovascular autoregulation using near-infrared spectroscopy, Stroke 38 (10) (2007) 2818-2825. 
[28] G. Themelis, H. D'Arceuil, S.G. Diamond, S. Thaker, T.J. Huppert, D.A. Boas, M.A. Franceschini, Near-infrared spectroscopy measurement of the pulsatile component of cerebral blood flow and volume from arterial oscillations, J. Biomed. Opt. 12 (1) (2007) 014033.

[29] H. Obrig et, A. Villringer, Beyond the visible- imaging the human brain with light, J. Cereb. Blood Flow Metab. 1-18 (2003).

[30] A. Malliani, Principles of Cardiovascular Neural Regulation in Health and Disease, Kluwer Academic Publishers, 2000.
[31] S. Boden, H. Obrig, C. Köhncke, H. Benav, S.P. Koch, J. Steinbrink, The oxygenation response to functional stimulation: Is there a physiological meaning to the lag between parameters? Neuroimage (2007) 100-107.

[32] D. Contini, A. Torricelli, A. Pifferi, L. Spinelli, R. Cubeddu, Proc. SPIE 6629 (2007) 662908. 